\title{
Typology, morphology and connectivity of pore space in claystones from reference site for research using BIB, FIB and cryo-SEM methods
}

\author{
G. Desbois ${ }^{1, a}$, J.L. Urai ${ }^{1}$, M.E. Houben ${ }^{1}$ and Y. Sholokhova ${ }^{1}$ \\ ${ }^{1}$ Structural geology, Tectonics and Geomechanics, RWTH Aachen University, Lochnerstrasse 4-20, D-52056 Aachen, Germany
}

\begin{abstract}
Detailed investigation of the morphology of the pore space in clay is a key factor in understanding the sealing capacity, coupled flows, capillary processes and associated deformation present in mudstones. Actually, the combination of ion milling tools (FIB and BIB), cryogenic techniques and SEM imaging offers a new alternative to study in-situ elusive microstructures in wet geomaterials and has the high potential to make a step change in our understanding of how fluids occur in pore space. By using this range of techniques, it is possible to quantify porosity, stabilize in-situ fluids in pore space, preserve the natural structures at nm-scale, produce high quality polished cross-sections for high resolution SEM imaging and reconstruct accurately microstructure networks in $3 \mathrm{D}$ by serial cross sectioning.
\end{abstract}

\section{Introduction}

In fluid-filled porous geomaterials, fluid-rock interactions have important effects on their physical and chemical properties. Though the bulk expressions of these properties are relatively well known for a number of materials, the relation between nanostructures and macroproperties are poorly understood for a complete understanding of the fluid-rock interactions. Because the porosity is intimately linked to coupled flows, capillary processes, permeability and associated deformation, a key factor to understanding these processes is a detailed understanding of the morphology of the pore space.

The characterization of porosity is especially relevant for mudrocks and clay-rich fault gouges since they are important mechanical elements in the Earth's crust and form seals for crustal fluids such as groundwater and hydrocarbons. Other fields of interest are the storage of anthropogenic carbon dioxide and radioactive waste in geologic formations.

There is a considerable body of literature on the characterization of porosity in clay-rich materials using many different techniques (metal injection porosimetry; magnetic susceptibility measurement; SEM; TEM; neutron scattering; NMR spectroscopy, CT scanner; ESEM...). However, the pore space characterization has been mostly indirect until now.

To investigate directly the meso-porosity (from $\mathrm{mm}$ to about ten $\mathrm{nm}$ in pore size) in mudstones, SEM imaging is certainly the most direct approach but, on the one hand, it is limited by the poor quality of the investigated surfaces (mainly broken or mechanically polished surfaces including the decoration of porosity by colored resin embedment), which make observation difficult, and the interpretation of the microstructures complicated; and on the other hand, most of conventional methods require dried samples in which the natural structure of pores could be damaged due to the desiccation and dehydration of the clay minerals. The recent development of ion milling tools (Broad Ion Beam, BIB and Focused Ion Beam, FIB) and cryo-SEM allows respectively producing exceptional high quality polished cross-sections suitable for high resolution porosity SEM imaging at nm-scale and investigating samples under wet conditions by cryogenic stabilization.

We report on a study of Boom clay from a reference site for research (Mol site, Belgium) using cryo-SEM at cryogenic temperature, with ion beam cross-sectioning to prepare smooth, damage free surfaces. Pores commonly have crack-like tips, preferred orientation parallel to bedding and power law size distribution. We define a number of pore types depending on shape and location in the microstructure. 3-D reconstruction by serial cross-sectioning shows 3-D connectivity of the pore space. These findings offer a new insight into the morphology of pores and quantification of porosity down to nano-scale and provide the basis for microstructure-based models of transport in clays.

\section{Samples and methods}

$\overline{\text { a. e-mail: g.desbois@ged.rwth-aachen.de }}$

This is an Open Access article distributed under the terms of the Creative Commons Attribution-Noncommercial License 3.0, which permits unrestricted use, distribution, and reproduction in any noncommercial medium, provided the original work is properly cited. 
We used undisturbed clay samples of Boom clay (Mol, Belgium, collected at depth $5.82 \mathrm{~m}-5.98 \mathrm{~m}$ above the HADES URF), which is potentially a good candidate for waste disposal. Samples were sealed in aluminium safety bags directly after the sampling to preserve samples against dehydration and oxidation. A comprehensive summary of physical and chemical properties of Boom clay is given in [1].

SEM imaging of pore space at high resolution in geosciences is on rapid development since ion beam milling tools have been demonstrated as a powerful technique to prepare smooth, damage free cross-sectioned surfaces at the state-of-the-art SEM resolution ([2], [3], [4], [5] and [6]). Two main types of ion source are available: (1) a broad ion beam (BIB, up to few mA, Argon source) is suitable to produce large polished cross-sections area of few $\mathrm{mm}^{2}$ (Figure 1.a), while the focused ion beam (FIB, 1pA - >50nA; Gallium source) is better used for fine and precise polished cross-sections areas of about few $\mathrm{m}^{2}$ (Figure 1.b). Because BIB is not focused, a shielding plate is placed on the top surface of the sample in order to create flat cross-section (Figure 1.a). Up to now, on the market, BIB cross sectioners are usually available as stand-alone machines able to produce single polished cross-section; while FIB cutters are now mainly integrated in SEM offering the possibility to perform serial cross sectioning suitable for 3D microstructures reconstruction (Figure 5, [2], [3], [4], [5] and [6]). However, the big advantage of BIB is that it is able to produce larger polished surfaces. This feature is of relevant interest for geosciences since it fits better to the typical size-range of microstructures and representative elementary area of geomaterials [3].
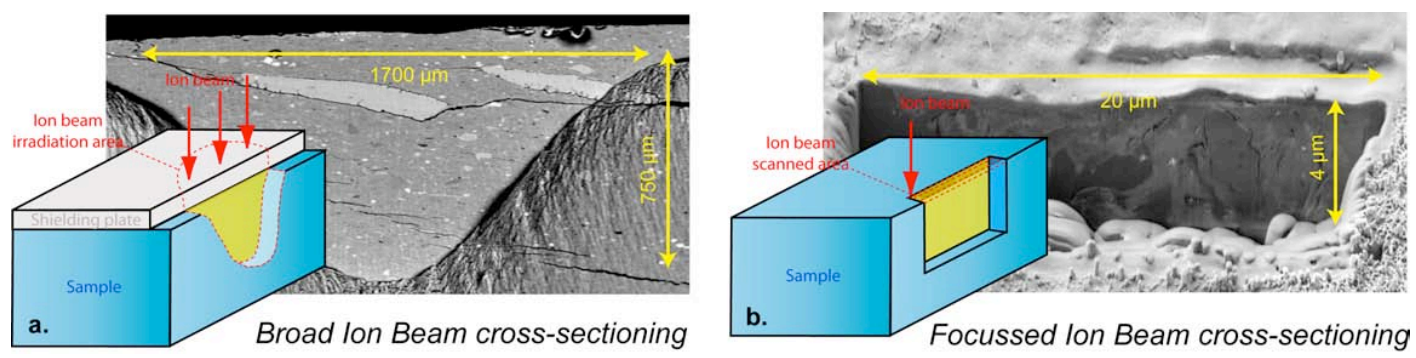

Fig. 1: Principles of ion beam cross-sectioning methods used to produce high quality polished surface suitable for high-resolution SEM imaging. (a) The Broad Ion Beam (BIB) method uses Argon gas source: the ion beam (about $500 \mu \mathrm{m}$ in diameter) irradiates the edge of sample un-masked by the shielding plate to guide the ion beam vertically to the top surface to create polished surface beneath the shielding plate. In background, a typical cross section performed by BIB on clay sample. (b) The Focused Ion Beam (FIB) method uses Gallium metal source. Because the beam is focused, shielding plate is not needed and the cross-section is produced by scanning the interesting region with ion beam. In background, a typical cross section performed by FIB on clay sample. By comparison with FIB, the polished area produced by BIB is larger of more than one order of magnitude for similar milling time.

In our experiments, we used a Zeiss FIB-cryo-SEM combination in NMI (Naturwissenschaftliches und Medizinisches Institut, Reutlingen, Germany) as described in Desbois et al. (2008), and a high resolution SEM and BIB at GFE, RWTH Aachen. Nitrogen was used to rapidly cool small samples (about $5 \times 5 \times 1 \mathrm{~mm}^{3}$ ) to stabilize by vitrification in-situ pore fluids. The FIB ion-milling tool located in the cryo-SEM chamber was used to prepare polished cross-sections and serial cross sectioning, prior to high resolution imaging of porosity by cryo-SEM. For comparison we studied polished cross-sections (about $2 \mathrm{~mm}^{2}$ ) from dried samples (at $\mathrm{T}=105-110^{\circ} \mathrm{C}$ ) prepared using a stand-alone BIB machine (cross-section polisher JEOL SM-09010; we used $6 \mathrm{kV}$ voltage, achieving currents of about $150-200 \mathrm{nA}$ ). Water content of our samples is $19-20 \mathrm{wt}$. \% (i.e. water content porosity around $36 \%$ ), in good agreement with the value in [1]; hence shrinkage during drying was around $10 \%$ in volume.

\section{Results and discussion}

\subsection{In-situ fluids imaging and freeze-drying process}

Figure 2 shows FIB-polished cross sections of shock-frozen wet samples. There is no visible damage and vitrified fluids appear as black phases filling pores. Fluids are clearly visible in largest pores located at the direct vicinity of non-clay minerals (Type III, see section 3.2). Imaging vitrified fluids in very small pores (i.e. those located in clay mineral aggregates; type II and III, see section 3.2 ) is problematic at the highest magnification since the energy of the beam sublimes the fluids before an image can be recorded.

As far as we know, this is the first time that fluids were directly imaged in in-situ conditions in mudrocks, offering new insights for the study of fluid- rock interaction (osmotic effect, ion exchange, pore alteration), especially if the BIB-FIB-cryo-SEM is coupled to chemical composition analysis tool (EDX, SIMS).

Cryo-stabilization is a fundamental step to stabilize pore microstructures without damage and to image the pore fluid. However, the presence of fluid in pores is not required to study the morphology of porosity because an empty pore provides better SE contrast and a 
3D view into the exposed pore. Hence, freeze-drying is the solution to remove frozen fluid phases from pores without damage since sublimation of frozen fluid phases involved in this process not allows the formation of ice crystals, which could damage the pore microstructures by volume expanding. Freeze-drying is performed by heating the vitrified sample after FIB sectioning, in the SEM chamber and under vacuum $\left(-130^{\circ} \mathrm{C}\right.$ to $-80^{\circ} \mathrm{C}$ during $20 \mathrm{~min}$., at $\left.8 \times 10^{-7} \mathrm{mbar}\right)$.
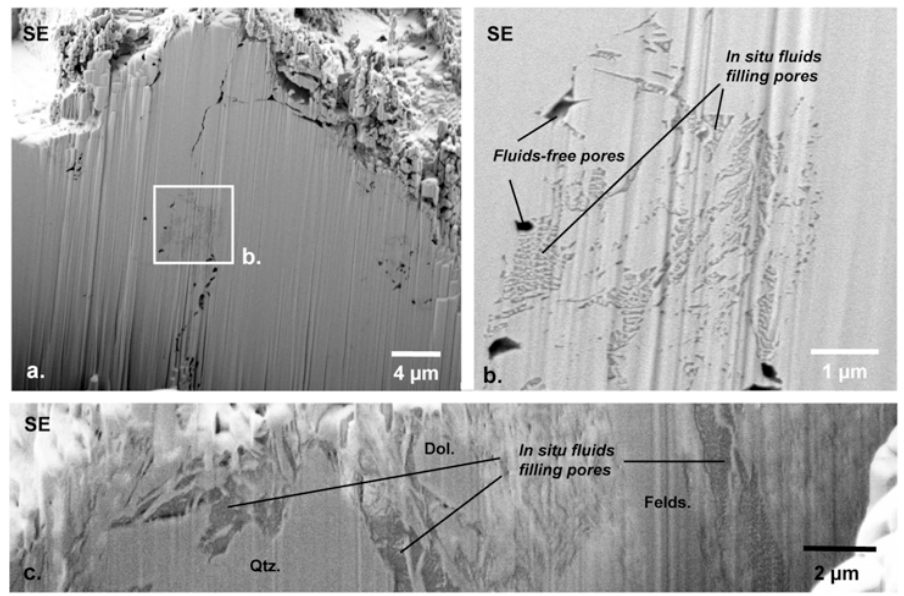

Fig. 2: SEM micrograph (SE) of FIB cross-section in Boom clay performed under cryo-condition. (a) overview of a FIB cross-section. (b) Details from (a) showing pores filled with in-situ frozen-fluid but also fluid-free pores. (c) In-situ fluids are clearly visible in the biggest pores. The biggest pores are located around the non-clay minerals $(\mathrm{Qtz}=$ Quartz, Dol. $=$ Dolomite., Felds. $=$ Feldspar $=$ K-feld $)$.

\subsection{Pore morphologies}

Figure 3 shows SE images of a dry sample sectioned by BIB. Drying and shrinkage reduce porosity, but we found no indication of changes in pore morphology by drying. Figure 3 gives unprecedented view of pores at pore scale.
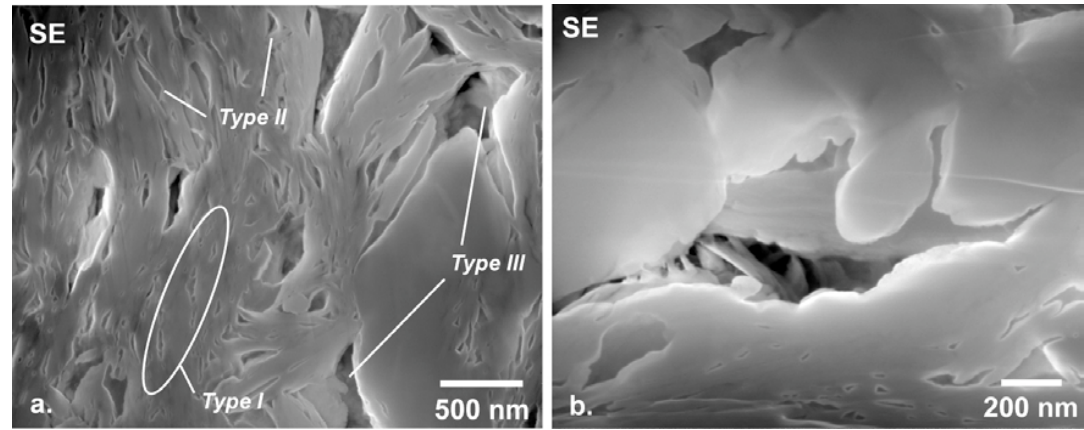

Fig. 3: SEM micrographs (SE) from BIB polished cross-sections performed on Boom clay. (a) The morphology of pores depends on clay particle arrangement and mineralogy. Type I - elongated pores between similarly oriented clay sheets, Type II - crescent-shaped pores in saddle reefs of folded sheet of clay and Type III - large jagged pores surrounding clast grains. (b) A pore at high magnification provides information on the morphology of the jagged pore walls and pore interiors and suggests the complex interconnectivity of pore space.

We distinguished 3 main types of pore morphology: (1) Type I- elongated pores between similarly oriented clay sheets, (2) Type IIcrescent-shaped pores in saddle reefs of folded sheet of clay and (3) Type III - large jagged pores surrounding clast grains. Type III 
pores are typically $>1$ um; Type II between $1 \mu \mathrm{m}-100 \mathrm{~nm}$ and Type I $<100 \mathrm{~nm}$, in agreement with the microstructural model of mudstones proposed by [7]. As shown in Figure 2.a, largest pores (type III) are located around clast grains while the smallest are preferentially located between clay particles (Type I and II). Pores are apparently oriented parallel to the bedding. Figure 3.b provides information on the morphology of the jagged pore walls and pore interiors. As far as can be seen in Figure 3, small pores are isolated while large pores show the pore throats connecting to neighboring pores. Pores commonly have sharp, crack-like tips.

The high slaking tendency of argillaceous rocks increases with the content in smectite content and with the presence of soluble minerals ([8]). We propose that the flat, crack-like pore morphology will induce high capillary forces in these pore- tips if a dry sample is rewetted, resulting in mechanical damage and destruction of the fabric by successive drying and wetting.

\subsection{Quantification of porosity}

BIB (Figure 4.a) and FIB- polished cross sections cut perpendicular to the bedding allow the distribution, orientation and shape of pores to be determined by imagery. BIB was used to prepare surface on dry samples whereas FIB was used on originally wet samples later freeze-dried. Noise was reduced and the images were threshold using Photoshop 8.0 followed by analysis using the ImageJ $1.38 \mathrm{x}-$ software ([9]). Objects less than 6 pixels in area (pixel dimension is $12.5 \mathrm{~nm}$ ) were interpreted as noise and not included in the analysis.

Results obtained for dry and wet sample are summarized in Figure 4 for a population of 1730 and 2320 pores respectively. For both, the distribution of pores is unimodal (Figure 4.c), $87 \%$ of the pores have an equivalent radius less than $100 \mathrm{~nm}$ and the total porosity is $26.3 \%$ in dry samples while it is $20.4 \%$ in wet samples. Pores with a radius less than $100 \mathrm{~nm}$ comprise $40 \%$ of the total porosity. The shape of the pores is mainly elongated (the average of form factor is 0.59 ) and orientation is close to the bedding (Figure 4.d). The porosity resolved in our images can be fitted by a power law $\left(\log \left(\mathrm{N}_{\mathrm{i}}\right)=-\mathrm{D} * \log \left(\mathrm{r}_{\mathrm{i}}\right)\right.$; where $\mathrm{N}_{\mathrm{i}}$ is the number of pores with the characteristic disk equivalent radius $r_{i}$ and $D$ the fractal dimension) for pore sizes between $r_{\min }=30 \mathrm{~nm}$ and $r_{\max }=125 \mathrm{~nm}$ (Figure 4.e). This suggests fractal scaling of the pore space in Boom clay. The power law exponent (i.e. fractal dimension D) is around 1.85, both for dry and wet samples.
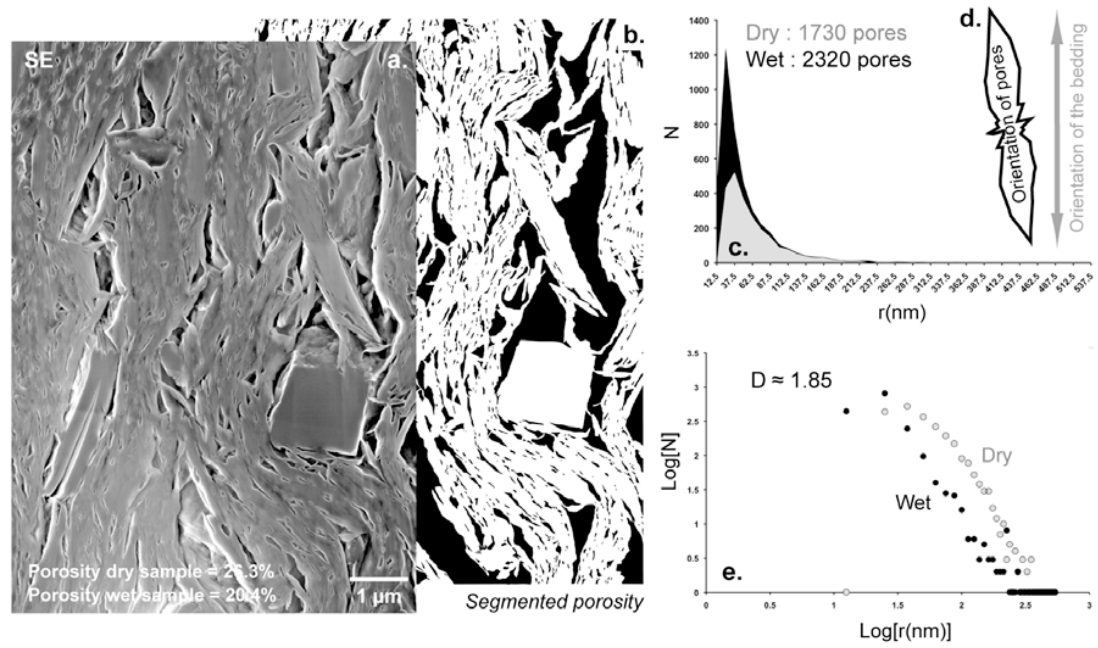

Fig. 4: Quantification of porosity from ion beam polished cross section. (a) Example of 2D apparent porosity of BIB cross section on dry Boom clay sample. (b) Segmentation of (a) needed for porosity image processing. (c) Pore size distribution in dry and wet samples. The pore size distribution is unimodal. $\mathrm{N}$ is the number of pore. (d) Orientation of pores compared with the orientation of the bedding. (e) Log-Log pore distribution curve for dry and wet samples. The porosity resolved in our images can be fitted by a power law corresponding to a fractal dimension of $\mathrm{D} \approx 1.85 \mathrm{for} 30<\mathrm{r}(\mathrm{nm})<125$.

For the fractal dimension of the 2-D apparent porosity we get similar results on dried and wet samples. In regards to results from figure 3.c, this suggests that the shrinkage affects the volume of pores but not their size distribution. The calculated fractal dimension $(D \approx 1.85)$ is only valid in the range between $30 \mathrm{~nm}$ and $125 \mathrm{~nm}$ : at short-length extremes, the data are limited by the microscope resolution and the picture's noise; at long-length extremes, the data are limited by the fact that the investigated area is not endlessly. Geological porous media ([10], [11], [12]) have been shown to have a fractal distribution of porosity. This paper is the first evidence that porosity in a clay material has a fractal distribution, and demonstrates the usefulness of our BIB/FIB-cryo-SEM technique for mesoporosity investigation in such rocks. 
Our SEM - measured porosity (20.4\% in freeze dried samples, $26.3 \%$ in dried samples) agrees quite well the porosity based on mercury porosimetry data (24-27\% in dried samples) for similar Boom clay samples though our measurement based on cryo-SEM experiment trends to give a lower porosity value. However, our SEM-measured porosity is much lower than the water content porosity $(36 \%$, [1]). Several reasons explain this discrepancy. First, the resolution of the SEM is about $10 \mathrm{~nm}$ although pores as small as $0.5 \mathrm{~nm}$ do exist as shown by other methods including mainly TEM. Extrapolation of the power law pore size distribution to values below the limit of resolution suggests that e.g. a sample with $20 \%$ resolved porosity has $2 \%$ porosity not resolved by this method. In addition, the tips of crack-shaped pores and the films of clay-bound water at grain boundaries and inside smectite grains are also not resolved fully; accounting for this gives another $8 \%$. Thus, although our approach is suitable for investigation of meso-porosity only, by making reasonable assumptions we can estimate the full porosity. However, for our freeze-dried sample this is still much lower than the water content porosity (36\%). In the dried sample, after correction for the unresolved pores and keeping in mind the possible artifacts produced by mercury injection such as pore collapse ([13], [14]) the porosity is in reasonable agreement with the water-content-porosity. We interpret this discrepancy to be due to the size of the analyzed samples. Mercury injection and water content measurement are typically performed on $\mathrm{cm}^{3}$ samples and the areas analyzed here are on the order of $10 \mu \mathrm{m}^{2}$. The size of a representative elementary volume for porosity in Boom clay is still to be determined, but future representative measurements will require study of larger sections.

It is well known that water content porosity in claystones does not reflect the effective porosity (i.e. the porosity accessible to freewater) because thin layers of clay-bound water cover the pore-walls ([15]). In our samples and based on segmented porosity, the evolution of the effective porosity in function of the bound-water layer thickness shows that increasing the thickness of bound-water layer results in decreasing of the effective porosity as a semi-linear function, which suggests that with increasing bound-water layer thickness pores usually do not segment into smaller ones. Following ([15], [16]) typical bound water is $2-6 \mathrm{~nm}$ thick. Thus, we estimated that the effective porosity represents between 90 and $70 \%$ of the porosity imaged in this contribution.

\subsection{Interconnectivity of pore space}

SEM imaging on large $\left(800 \times 260 \mu^{2}\right)$ BIB polished cross-sections on dried Boom clay samples (cut perpendicular to bedding) shows a strong anisotropy of the pore shapes, the pore network being mainly connected parallel to the bedding. In order to study the undisturbed wet pore space in 3-D, a serial cross-sectioning procedure was performed using the FIB-cryo-SEM. Resulting slices are $500 \mathrm{~nm}$ thick. Figure 5 shows the reconstruction of the porosity in depth (over $7 \mu \mathrm{m}$ ) around a quartz grain. It confirms the strong connectivity anisotropy observed in dried samples and gives direct evidence that the largest pores surrounding the quartz grains form a connected "backbone" of $100 \mathrm{~nm}$ to several microns thick mainly oriented parallel to bedding. Interconnectivity is difficult to analyze for the smallest pores because of aliasing (the current distance between slices is $500 \mathrm{~nm}$, but this can be reduced to less than $20 \mathrm{~nm}$ ).

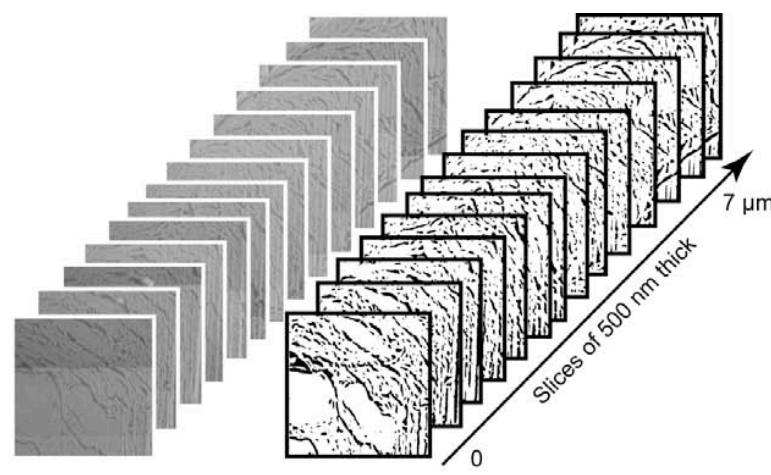

a. Serial FIB

b. Segmentation

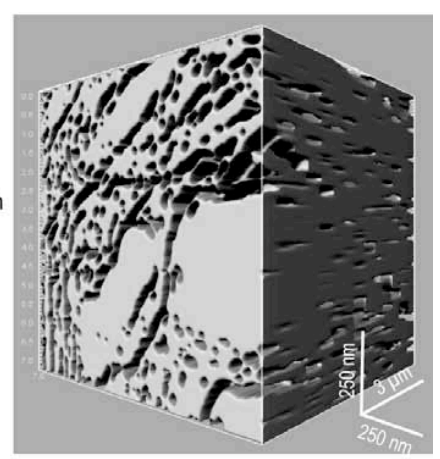

c. $3 D$ reconstruction

Fig. 5: FIB serial cross-sections performed under cryo-condition in the neighborhood of two quartz grains in Boom-clay, perpendicular to the bedding. The slice thickness is $500 \mathrm{~nm}$. (a) Initial SE pictures and, (b) segmented pictures and (c) the reconstructed 3D pore network was generated using Bitplane software for a preview purpose. The porosity is mainly connected along the direction parallel to the bedding.

The calculated fractal dimension $(D \approx 1.85)$ is close to the fractal dimension of the Sierpinsky's carpet $(\mathrm{D}=1.89)$. In [12], it is suggested that soils may be modeled by the Menger's sponge $(\mathrm{D}=2.73)$, which is the 3-D version of the Sierpinsky's carpet. Unfortunately, the observation of the strong anisotropy of the pore distribution and the interconnectivity (Fig. 5) does not corroborate this simple suggestion for Boom-clay. 
The reduction of the slice thickness (down to $20 \mathrm{~nm}$ ) will produce high-resolution models of pore space with the possibility to model fluid flow and microstructure-based models of transport in clays ([17], [18]). This will provide a unique opportunity to model the flow through in-situ pore network, study the in-situ effective interconnectivity and verify the viability of our proposed clay fractal model.

\section{Conclusions and further developments}

Application of the BIB-FIB-cryo-SEM methods to study the porosity in clay-rich materials is clearly relevant for waste disposal, hydrocarbon production, basin modelling, fault zone studies and others. It has the potential to open a new field of investigations, producing an atlas of accurate pore models of mudstones. These methods offer a powerful combination for direct and in-situ investigations of the elusive structures in rich-clay materials at pore scale opening a new field of investigations to study relation between nanostructures and macro-properties which are poorly understood at present. As far as we know, this is the first time that fluids were directly imaged in in-situ conditions in mudrocks, offering new insights for the study of fluid- rock interaction (osmotic effect, ion exchange, pore alteration). The reduction of the slice thickness (down to $20 \mathrm{~nm}$ ) for serial sectioning will produce high-resolution models of pore space with the possibility to model fluid flow and microstructure-based models of transport in clays. This will provide a unique opportunity to model the flow through in-situ pore network (Lattice Boltzmann method, [19]), study the effective interconnectivity, investigate the natural fluid distribution and quantify investigated microstructures ([3] and [4]).

The next generation of ion beam-cryo-SEM instrument, especially designed for geomaterials, will probably include BIB source instead of FIB. The first BIB-cryo-SEM machine is currently being installed at RWTH Aachen University in Germany (Deutsche Forschungsgemeinschaft - Project UR 64/9-2) and will be dedicated to micro imaging of elusive microstructures in wet geomaterials.

\section{Acknowledgement}

We thank Christoph Nussbaum and Mieke de Craen of SCK-CEN for help with obtaining the Boom clay samples. We are grateful to Claus Burkhardt (NMI at university of Tübingen, Reutlingen, Germany), Alexander Schwedt (GFE at RWTH, Aachen University, Aachen, Germany) for their technical support for the SEM imaging and Jochen Hürtgen for image processing. This project was funded by the Deutsche Forschungsgemeinschaft (UR 64/9-2).

\section{References}

1. J.Y. Boisson,: Clay Club Catalogue of Characteristics of Argillaceous Rocks, OECD/NEA/RWMC/IGSC (Report NEA no. 4436, OECD/NEA Paris, France, p. 72, 2005)

2. G. Desbois, J.L. Urai and P.A. Kukla, e-Earth, 4, 1-8 (2009)

3. G. Desbois, J.L. Urai, C. Burkhardt, M.R. Drury, M. Hayles, and B. Humbel, Geofluids, 8, 60-72 (2008)

4. L. Holzer, Ph. Gasser, A. Kaech, M. Wegmann, A. Zingg, R. Wepf and B. Münch, Journal of Microscopy, 227, 216-228 (2007)

5. L. Holzer, B. Münch, M. Rizzi, R. Wepf, P. Marschall and T. Graule, Applied Clay Sciences, (In review)

6. D.A.M. De Winter, C.T.W.M. Schneijdenberg, M.N. Lebbink, B. Lich, A.J. Verkleij, M.R. Drury and B.M. Humbel, Journal of Microscopy - Oxford, 233, 372-383 (2009)

7. A. Hildenbrand, B. M. Krooss, and J. L Urai, IUTAM Symposium on Physicochemical and Electromechanical Interactions in Porous Media, 125, 231-237 (2005)

8. I. A. Sadisun, H. Shimada, M. Ichinose, and K. Matsui, Geotechnical and Geological Engineeering, 23, 199-218 (2005)

9. M.D. Abramoff, P.J. Magelhaes and S.J. Ram, Biophotonics, International, 11, 36-42 (2004)

10. G. Daccord and R. Lenormand, Nature, 325, 41- 43 (1987)

11. C. E. Krohn and A. H. Thompson, Phys. Rev., B33, 6366-6374 (1986)

12. M. Rieu and G. Sposito, Soil Sciences Society of America, 55, 1239-1244 (1991)

13. A. Hildenbrand and J.L. Urai, Marine and Petroleum Geology, 20, 1185-1200 (2003)

14. J. Majling, S. Komarneni, and V. S. Fajnor, Journal of porous materials, 1, 91-95 (1995)

15. T. Saarenketo, Journal of Applied Geophysics, 40, 73-88 (1998)

16. Y. Nakashima and F. Mitsumori, Applied Clay Sciences, 28, 209-221 (2005)

17. J.T. Fredrich and W.B. Lindquist, International journal of rock mechanics and mining sciences, 34, 3-4 (1997)

18. P.D.D. Bons, D. Koehn, and M.W. Jessell, Microdynamics Simulation (Series: Lecture Notes in Earth Sciences, 106, p. 406, 2008)

19. Y. Sholokhova, D. Kim and W.B. Lindquist. Advances in Water Ressources, 32, 205-212 (2009) 